\title{
Type 2 diabetes is associated with failure of non-operative treatment for sternoclavicular joint infection
}

\author{
Shriya B. Reddy ${ }^{1}$, Jack Mizelle ${ }^{1}$, Helene M. Sterbling ${ }^{2}$, Brenda Lin ${ }^{3}$, Virginia R. Litle ${ }^{1,3,4}$, Kei Suzuki ${ }^{1,3,4}$ \\ ${ }^{1}$ Boston University School of Medicine, Boston, MA, USA; ${ }^{2}$ Inova Fairfax Hospital, Falls Church, VA, USA; ${ }^{3}$ Department of Surgery, Boston Medical \\ Center, Boston, MA, USA; ${ }^{4}$ Department of Surgery, Division of Thoracic Surgery, Boston Medical Center, Boston, MA, USA \\ Contributions: (I) Conception and design: SB Reddy, HM Sterbling, VR Litle, K Suzuki; (II) Administrative support: B Lin, VR Litle, K Suzuki; (III) \\ Provision of study materials or patients: HM Sterbling, K Suzuki; (IV) Collection and assembly of data: SB Reddy, J Mizelle, HM Sterbling; (V) Data \\ analysis and interpretation: SB Reddy, HM Sterbling, B Lin, K Suzuki; (VI) Manuscript writing: All authors; (VII) Final approval of manuscript: All \\ authors. \\ Correspondence to: Kei Suzuki, MD. 88 E. Newton Street, Robinson 7308, Boston, MA 02118, USA. Email: kei.suzuki@bmc.org.
}

Background: A standardized treatment algorithm for sternoclavicular joint infection management is lacking in the literature. While major risk factors for sternoclavicular joint infection, including immunosuppression, rheumatoid arthritis, type 2 diabetes, indwelling catheters, and intravenous drug use have been identified, clear association with treatment outcome has not been established. As our safety net hospital treats a patient population with high incidence of intravenous drug use, we sought to identify risk factors associated with failure of non-operative management of sternoclavicular joint infection.

Methods: We conducted a retrospective cohort study, reviewing charts of patients diagnosed with sternoclavicular joint infection between January 2001 and December 2017 to collect demographic information as well as clinical risk factors and treatment patterns. A chi-square test was performed to determine any association between clinical variables and management, as well as relation to treatment outcome.

Results: The study cohort consisted of 35 patients with diagnosis of sternoclavicular joint infection and complete follow-up. Intravenous drug use was prevalent, seen in $45.6 \%$ (16/35) of subjects, though there was no association with failure of non-operative management $(\mathrm{P}=0.50)$. Operative management was the initial treatment for $25.7 \%(9 / 35)$ of subjects and was associated with abscess on presentation $(\mathrm{P}=0.03)$. Failure of non-operative management was seen in $26.9 \%$ (7/26). Type 2 diabetes was associated with failed initial nonoperative management, present in $42.9 \%(3 / 7)$ of patients $(\mathrm{P}=0.03)$ experiencing failure.

Conclusions: This study constitutes the largest series of sternoclavicular joint infection with intravenous drug use. While intravenous drug use was not associated with failure of non-operative management, we observed that type 2 diabetes is associated with failure of non-operative management and could be considered in determining management of sternoclavicular joint infection patients.

Keywords: Sternoclavicular joint infection (SCJi); type 2 diabetes; non-operative management

Submitted May 17, 2020. Accepted for publication Sep 07, 2020.

doi: $10.21037 /$ jtd-20-1897

View this article at: http://dx.doi.org/10.21037/jtd-20-1897

\section{Introduction}

Septic arthritis due to sternoclavicular joint infection (SCJi) composes approximately $1 \%$ of all infectious arthritis cases in the general population (1). Several comorbidities have been reported as predisposing factors for SCJi: intravenous drug use (IVDU), human immunodeficiency virus (HIV), diabetes mellitus II (DM2), prior chest trauma, presence of indwelling catheter, renal failure, rheumatoid arthritis, and immunosuppression (1-3). A standardized treatment algorithm for SCJi management is lacking in the literature. 
Management strategies include non-operative treatment with extended antibiotics, or may range from incision and drainage (I\&D) to operative open joint resection requiring flap closure or negative wound pressure therapy (1,4-6).

Though IVDU has been implicated as a major comorbidity, its association with outcomes of non-operative management has not been studied. As a high-burden safety net hospital with prevalent IVDU among our patients, there exists a need to identify appropriate treatment approach based on risk factors. We hypothesized that non-operative management of SCJi in IVDU patients would be more likely to fail than in non-IVDU patients, and sought to identify other risk factors associated with treatment failure.

We present the following article in accordance with the STROBE reporting checklist (available at http://dx.doi. org/10.21037/jtd-20-1897).

\section{Methods}

The study was conducted in accordance with the Declaration of Helsinki (as revised in 2013). The study was approved by the Boston University School of Medicine and Boston Medical Center (BMC) Institutional Review Board (IRB) (No. FWA00000301) and individual consent for this retrospective analysis was waived. A retrospective chart review was conducted of all patients who underwent treatment for SCJi at BMC between January 2001 and December 2017. These charts were obtained via a textbased search within the BMC Clinical Data Warehouse using the following key words: "sternoclavicular (joint) + infection", "sternoclavicular (joint) + osteomyelitis", "sternoclavicular + septic joint", and "sternoclavicular (joint) + septic arthritis". Inclusion criteria for the study cohort were diagnosis of osteomyelitis or septic SCJ following positive culture or imaging findings. Medical records of all patients were reviewed for demographic identifiers, including age at diagnosis, gender, insurance status, and body mass index (BMI), as well as common risk factors: IVDU, HIV, DM2, chest trauma, indwelling catheter, renal failure, rheumatoid arthritis, and immunosuppression. Patient-reported symptoms such as erythema, pain, and swelling were identified, as well as more objective signs of quantified fever, leukocytosis or leukopenia. Pertinent findings from imaging modalities and cultures were collected.

Subsequent diagnoses and details of SCJi management were recorded. Non-operative treatment included imageguided aspiration and extended (6-week) intravenous antibiotic therapy, while operative management options consisted of antibiotics in addition to curettage, resection, or open drainage. The primary outcome of interest was success of treatment, defined by lack of further intervention and no recurrence of infection. Other outcome measures included patient compliance, functional impairment, adverse events of treatment, 30-day readmission, and mortality, assessed at primary care follow up appointment.

\section{Statistical analysis}

Statistical analysis consisted of chi-square analysis to test for association between demographic variables, risk factors, presenting signs and symptoms, treatment modality, as well as outcomes of operative vs. non-operative treatments. Differences were considered statistically significant at a $\mathrm{P}$ value $<0.05$. All analyses were performed using IBM SPSS Statistics, version 25 (IBM Corp., Armonk, NY, USA).

\section{Results}

A total of 42 patients were initially identified for the study, with seven excluded for lack of outpatient follow-up; study population consisted of 35 patients. Demographics of the study group are summarized in Table 1. Median age and BMI of the study cohort were 51 years (Range: 18-88 years; Q1: 41 years; Q3: 60 years) and 26.5, respectively. Federally-funded medical coverage via Medicaid and Medicare was provided for $54.3 \%$ (19/35) of patients. IVDU was prevalent, seen in $45.7 \%$ (16/35) of patients with SCJi.

Management modalities for the study cohort and outcomes are shown in Figure 1 . Non-operative management was the initial treatment for $74.3 \%(26 / 35)$ of patients. Of these, $11.5 \%(3 / 26)$ additionally underwent percutaneous joint drainage. Operative management was the initial treatment of choice for $25.7 \%(9 / 35) ; 33.3 \%(3 / 9)$ were performed with immediate closure with pectoralis flap. Resolution occurred in $88.9 \%$ (8/9). All patients managed operatively underwent surgical resection at the SCJ.

Association of risk factors with treatment modality is shown in Table 2. Finding of abscess on presentation was associated with initial operative management $(\mathrm{P}=0.03)$. Other symptoms, demographic variables, and comorbidities were not significantly associated with choice of initial treatment. Association of IVDU with initial non-operative management approached significance $(\mathrm{P}=0.10)$, with $53.8 \%$ $(14 / 26)$ of patients treated non-operatively on original 
Table 1 Patient demographics and clinical characteristics

\begin{tabular}{|c|c|}
\hline Patient variables & $\%(n)$ \\
\hline \multicolumn{2}{|l|}{ Demographics } \\
\hline Median age (y) & 51 \\
\hline Median BMI & 26.5 \\
\hline \multicolumn{2}{|l|}{ Gender } \\
\hline Male & $65.7 \%(23 / 35)$ \\
\hline Female & $34.3 \%(12 / 35)$ \\
\hline \multicolumn{2}{|l|}{ Insurance status } \\
\hline Medicaid & $34.3 \%(12 / 35)$ \\
\hline Medicare & $20.0 \%(7 / 35)$ \\
\hline Private/commercial & $14.3 \%(5 / 35)$ \\
\hline \multicolumn{2}{|l|}{ Risk factor } \\
\hline Chest trauma & $5.7 \%(2 / 35)$ \\
\hline DM2 & $25.7 \%(9 / 35)$ \\
\hline CKD & $22.9 \%(8 / 35)$ \\
\hline HIV & $2.9 \%(1 / 35)$ \\
\hline Immunosuppression & $5.7 \%(2 / 35)$ \\
\hline Indwelling catheter & $28.6 \%(10 / 35)$ \\
\hline IVDU & $45.7 \%(16 / 35)$ \\
\hline
\end{tabular}

BMI, body mass index; DM2, diabetes mellitus type 2; CKD, chronic kidney disease; HIV, human immunodeficiency virus; IVDU, intravenous drug user.

presentation having comorbid IVDU, compared to $22.2 \%$ (2/9) patients who underwent operative management and reported IVDU. In $97.1 \%$ (34/35) of the study cohort, imaging in the form of CT or MRI was performed. There was no difference between the study groups as far as the type of imaging obtained. CT or MRI was performed in $96.1 \%(25 / 26)$ of non-operative group and $100 \%(9 / 9)$ of the operative group. CT was performed in $84.6 \%(22 / 26)$ of non-operative group and $88.9 \%(8 / 9)$ of the operative group. MRI was performed in $50.0 \%(13 / 26)$ of the nonoperative group and $55.6 \%(5 / 9)$ of the operative group.

Of the 26 patients managed non-operatively, $73.1 \%$ $(19 / 26)$ were treated successfully while treatment failure was observed in $26.9 \%(7 / 26)$. When evaluating factors associated with success of non-operative treatment, neither age $(\mathrm{P}=0.56)$ nor gender $(\mathrm{P}=0.36)$ was statistically significant. Presenting signs and symptoms, including a finding of abscess, also were not associated with failure of non-operative management (Table 3). DM2 was seen in $23.1 \%(6 / 26)$ and was significantly associated with failure of non-operative management. Those with DM2 composed only $15.8 \%(3 / 19)$ of patients experiencing success with non-operative management, compared to $42.9 \%(3 / 7)$ experiencing failure $(\mathrm{P}=0.03)$.

Bacteremia was diagnosed in $71.4 \%$ (25/35) of patients, though it had no association with initial management plan $(\mathrm{P}=0.51)$ or outcome of conservative treatment $(\mathrm{P}=0.20)$. Blood cultures of bacteremic patients grew Methicillinresistant Staphylococcus aureus (MRSA) in 20\% (5/25) and Methicillin-sensitive Staphylococcus aureus (MSSA) in 48\% $(12 / 25)$ of cases; other organisms speciated on culture included Streptococcus agalactiae, Pseudomonas aeruginosa, Propionibacterium acnes, and Mycobacterium kansasii. No association was found between presence of MRSA and failure of non-operative treatment even after adjustment for population size $(\mathrm{n}=3, \mathrm{P}=0.17)$. $S$. aureus bacteremia, composing $68 \%(17 / 25)$ of cases and inclusive of both MRSA and MSSA, was also found to be unrelated to failure of non-operative treatment $(\mathrm{P}=0.39)$.

Of those who failed treatment with an initial six-week course of antibiotics, $42.9 \%$ (3/7) were readmitted for worsening osteomyelitis or secondary endocarditis, with a subsequent $57.1 \%$ (4/7) undergoing open joint resection and additional antibiotic therapy. One patient with failed medical management underwent secondary percutaneous joint drainage without supplemental antibiotics. The remaining $28.6 \%(2 / 7)$ received a repeat course of antibiotics. Noncompliance with antibiotics was noted in only one of the patients with failure of initial non-operative management. Overall compliance with medical treatment was found to be $97.1 \%(34 / 35)$.

\section{Discussion}

Infection of the sternoclavicular joint is a rare condition with high morbidity. As there is no guideline in deciding the initial management between conservative versus surgical, treatment of SCJi is variable. Commonly, initial management is guided by imaging findings. Two treatment algorithms have been proposed in the literature for standardization of SCJi management, both with notable prioritization of imaging findings on decision-making. Per an algorithm detailed by Abu Arab et al., SCJi with mild signs of inflammation on imaging should be conservatively managed with antibiotics, only proceeding to the operating room for I\&D if unsuccessfully resolved. Signs of abscess 


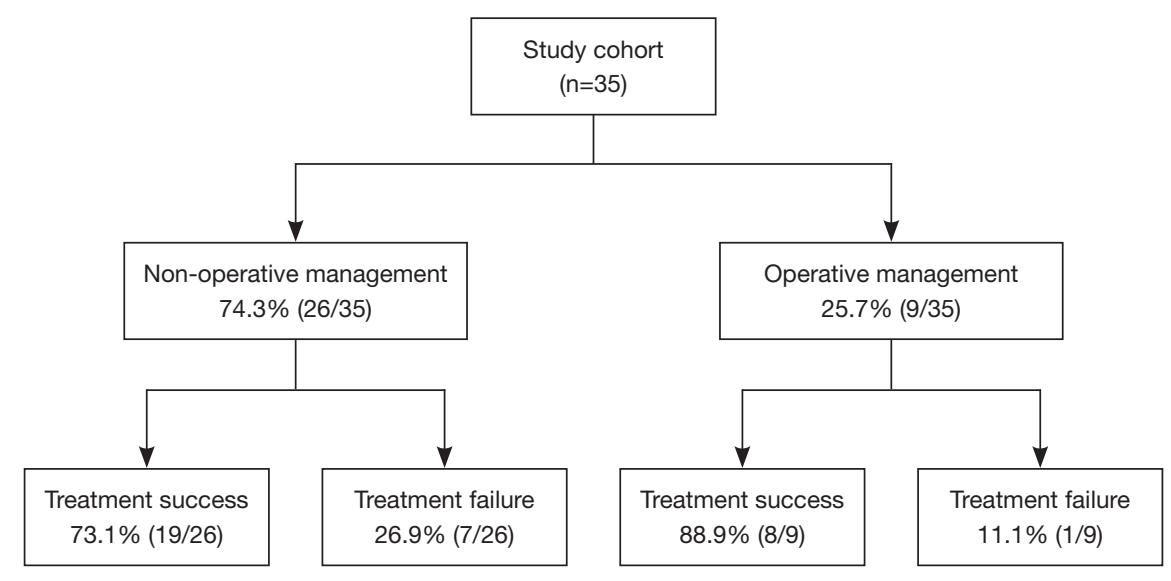

Figure 1 Study cohort.

without osteomyelitis should prompt I\&D, with failed cases proceeding to curettage. Abscess with concurrent osteomyelitis should be treated initially with both I\&D and curettage, with escalation to surgical resection of SCJ only in cases of severe osteomyelitis and recurrent infection (7). Alternatively, a multi-institutional study conducted by Murga et al., favors early joint debridement in all cases with positive imaging findings, ranging from fluid collection to osteomyelitis, in addition to early broad-spectrum antibiotics with MRSA coverage (1). Our own findings appear to align with this image-guided management, as all but one patient in the study received either a CT or MRI, and presenting sign of abscess was associated with initial operative management in our cohort.

While imaging findings appear to provide some guidance in deciding initial management of SCJi, we sought to explore the significance of other clinical factors in the current study. The United States has seen an increase in opioid use disorder and related hospitalizations in recent years, as reported by the National Heroin Task Force. Injection drug use in particular, both intravenous and intramuscular, is implicated as a major route of use portending poor patient outcomes due to bloodborne infection (8). At BMC, a 2012 study by Walley et al. reports approximately $17.5 \%$ of inpatients have diagnosed substance use disorder (9). Since our institution is a tertiary safety-net hospital, we initially sought to investigate whether IVDU is associated with a high failure rate of conservative management. A review of 180 cases of sternoclavicular septic arthritis published by Ross et al. described IVDU in 21\% (36/170) of patients and DM2 in 13\% (22/170). In comparison, our study cohort demonstrated higher prevalence of IVDU, with nearly half of the study population reporting active IVDU. We also demonstrated higher presence of DM2, occurring in 25.7\% of the patient cohort (9/35). To our knowledge, our study has the highest published composition of IVDU among SCJi patients (Table 4). Despite the high IVDU prevalence and our hypothesis that it would be associated with failure rate of conservative management, we did not find this association in our study. Instead, our results suggest that concomitant IVDU and SCJi can be observed in high incidence without measurable impact on patient outcome.

When comparing patients successfully treated with non-operative management of SCi to those who failed conservative therapy, our study uniquely identified DM2 as a predisposing factor for the failure of antibiotic treatment alone. Other published risk factors for failure of conservative management tend to be related to immunocompromised status. Aside from DM2, however, we did not find factors such as HIV, chronic kidney disease, and other immunocompromised states to be associated with non-operative treatment failure. Still, DM2 is considered to contribute to mechanisms of immunosuppression, increasing susceptibility of diabetic patients to infectious diseases, which often present with increased severity (12). Reported data demonstrates a risk ratio of 2.17 for infection-related hospitalization and of 1.92 for infectionrelated deaths in diabetic patients compared to non-diabetic controls (13).

Additionally, in the review by Ross et al., of the $42 \%$ (72/170) of patients treated conservatively with long-term antibiotics, $15.3 \%$ (11/72) failed to resolve. The majority, $60 \%(102 / 170)$, underwent initial surgical management (2). In comparison, our study had a low number of patients 
Table 2 Factors associated with initial treatment modality

\begin{tabular}{|c|c|c|c|}
\hline Variable & $\begin{array}{l}\text { Non-operative } \\
\quad(n=26)\end{array}$ & $\begin{array}{l}\text { Operative } \\
\qquad(n=9)\end{array}$ & $P$ value \\
\hline \multicolumn{4}{|l|}{ Demographic variable } \\
\hline Age $<51$ y & $46.2 \%(12 / 26)$ & $33.3 \%(3 / 9)$ & 0.39 \\
\hline \multicolumn{4}{|l|}{ Gender } \\
\hline Male & $73.1 \%(19 / 26)$ & $44.4 \%(4 / 9)$ & 0.13 \\
\hline Female & $26.9 \%(7 / 26)$ & $55.6 \%(5 / 9)$ & \\
\hline \multicolumn{4}{|c|}{ Presenting sign or symptom } \\
\hline Erythema & $34.6 \%(9 / 26)$ & $11.1 \%(1 / 9)$ & 0.18 \\
\hline Pain & $84.6 \%(22 / 26)$ & $77.8 \%(7 / 9)$ & 0.49 \\
\hline Swelling & $69.2 \%(18 / 26)$ & $66.7 \%(6 / 9)$ & 0.60 \\
\hline Abscess & $34.6 \%(9 / 26)$ & $77.8 \%(7 / 9)$ & $0.03^{*}$ \\
\hline Fever & $42.3 \%(11 / 26)$ & $22.2 \%(2 / 9)$ & 0.25 \\
\hline $\begin{array}{l}\text { Leukocytosis/ } \\
\text { Leukopenia }\end{array}$ & $38.5 \%(10 / 26)$ & $22.2 \%(2 / 9)$ & 0.32 \\
\hline \multicolumn{4}{|l|}{ Clinical risk factor } \\
\hline IVDU & $53.8 \%(14 / 26)$ & $22.2 \%(2 / 9)$ & 0.10 \\
\hline $\mathrm{HIV}+$ & $3.8 \%(1 / 26)$ & $0 \%(0 / 9)$ & 0.74 \\
\hline DM2 & $23.1 \%(6 / 26)$ & $33.3 \%(3 / 9)$ & 0.42 \\
\hline Chest trauma & $3.8 \%(1 / 26)$ & $11.1 \%(1 / 9)$ & 0.45 \\
\hline Indwelling catheter & $26.9 \%(7 / 26)$ & $33.3 \%(3 / 9)$ & 0.51 \\
\hline Renal failure & $23.1 \%(6 / 26)$ & $22.2 \%(2 / 9)$ & 0.67 \\
\hline Immunosuppressants & $7.7 \%(2 / 26)$ & $0 \%(0 / 9)$ & 0.55 \\
\hline \multicolumn{4}{|l|}{ Imaging } \\
\hline CT & $84.6 \%(22 / 26)$ & $88.9 \%(8 / 9)$ & 0.61 \\
\hline MRI & $50.0 \%(13 / 26)$ & $55.6 \%(5 / 9)$ & 0.54 \\
\hline Either CT or MRI & $96.1 \%(25 / 26)$ & $100 \%(9 / 9)$ & 0.74 \\
\hline
\end{tabular}

CT, computed tomography; DM2, diabetes mellitus type 2; HIV, human immunodeficiency virus; IVDU, intravenous drug user; $\mathrm{MRI}$, magnetic resonance imaging. ${ }^{*} \mathrm{P}<0.05$.

treated with initial surgical management, only $25.7 \%$. Although several previous studies widely consider surgical source control to be the optimal management (4-6), we found no significant difference in failure rate of conservative treatment in patients without comorbid DM2. In those with bacteremia in the review by Ross et al., S. aureus was the causative organism in 49\% (86/170) of isolates. Interestingly, a study recently published by Jang et al. commented on $100 \%(11 / 11)$ resolution in patients
Table 3 Factors associated with failure of initial non-operative management

\begin{tabular}{|c|c|c|c|}
\hline Variables & $\begin{array}{l}\text { Success rate } \\
\quad(n=19)\end{array}$ & $\begin{array}{l}\text { Failure rate } \\
\qquad(n=7)\end{array}$ & $P$ value \\
\hline \multicolumn{4}{|l|}{ Demographic variable } \\
\hline Age $<51$ y & $36.8 \%(7 / 19)$ & $42.9 \%(3 / 7)$ & 0.56 \\
\hline \multicolumn{4}{|l|}{ Gender } \\
\hline Male & $73.7 \%(14 / 19)$ & $57.1 \%(4 / 7)$ & 0.36 \\
\hline Female & $26.3 \%(5 / 19)$ & $42.9 \%(3 / 7)$ & \\
\hline \multicolumn{4}{|c|}{ Presenting sign or symptom } \\
\hline Erythema & $26.3 \%(5 / 19)$ & $57.1 \%(4 / 7)$ & 0.16 \\
\hline Pain & $89.5 \%(17 / 19)$ & $71.4 \%(5 / 7)$ & 0.29 \\
\hline Swelling & $68.4 \%(13 / 19)$ & $71.4 \%(5 / 7)$ & 0.59 \\
\hline Abscess & $36.8 \%(7 / 19)$ & $28.6 \%(2 / 7)$ & 0.54 \\
\hline Fever & $31.6 \%(6 / 19)$ & $71.4 \%(5 / 7)$ & 0.08 \\
\hline $\begin{array}{l}\text { Leukocytosis/ } \\
\text { leukopenia }\end{array}$ & $36.8 \%(7 / 19)$ & $42.9 \%(3 / 7)$ & 0.56 \\
\hline \multicolumn{4}{|l|}{ Clinical risk factor } \\
\hline IVDU & $52.6 \%(10 / 19)$ & $42.9 \%(3 / 7)$ & 0.50 \\
\hline $\mathrm{HIV}+$ & $10.5 \%(2 / 19)$ & $0 \%(0 / 7)$ & 0.53 \\
\hline DM2 & $15.8 \%(3 / 19)$ & $42.9 \%(3 / 7)$ & $0.03^{*}$ \\
\hline Indwelling catheter & $26.3 \%(5 / 19)$ & $28.6 \%(2 / 7)$ & 0.64 \\
\hline Renal failure & $21.1 \%(4 / 19)$ & $28.6 \%(2 / 7)$ & 0.53 \\
\hline Immunosuppression & $5.3 \%(1 / 19)$ & $14.3 \%(1 / 7)$ & 0.47 \\
\hline
\end{tabular}

DM2, diabetes mellitus type 2; HIV, human immunodeficiency virus; IVDU, intravenous drug user. ${ }^{*} \mathrm{P}<0.05$.

diagnosed with S. aureus-implicated SCJi and treated with optimal medical management (10). Our study had higher prevalence of $S$. aureus bacteremia compared to that reported by Ross et al., but without any significant association with failure of non-operative treatment.

In our cohort, non-compliance with extended antibiotics was identified in one patient who failed non-operative management. Notably, those patients who did fail medical management were at risk for developing complications including endocarditis and chronic osteomyelitis. Secondary operative management following this initial treatment failure typically involved joint resection, a more invasive surgery that should be reserved for serious infection as suggested by Abu Arab et al. (7). As such, identification of patients diagnosed with SCJi and concurrent DM2 for 
Table 4 Literature review

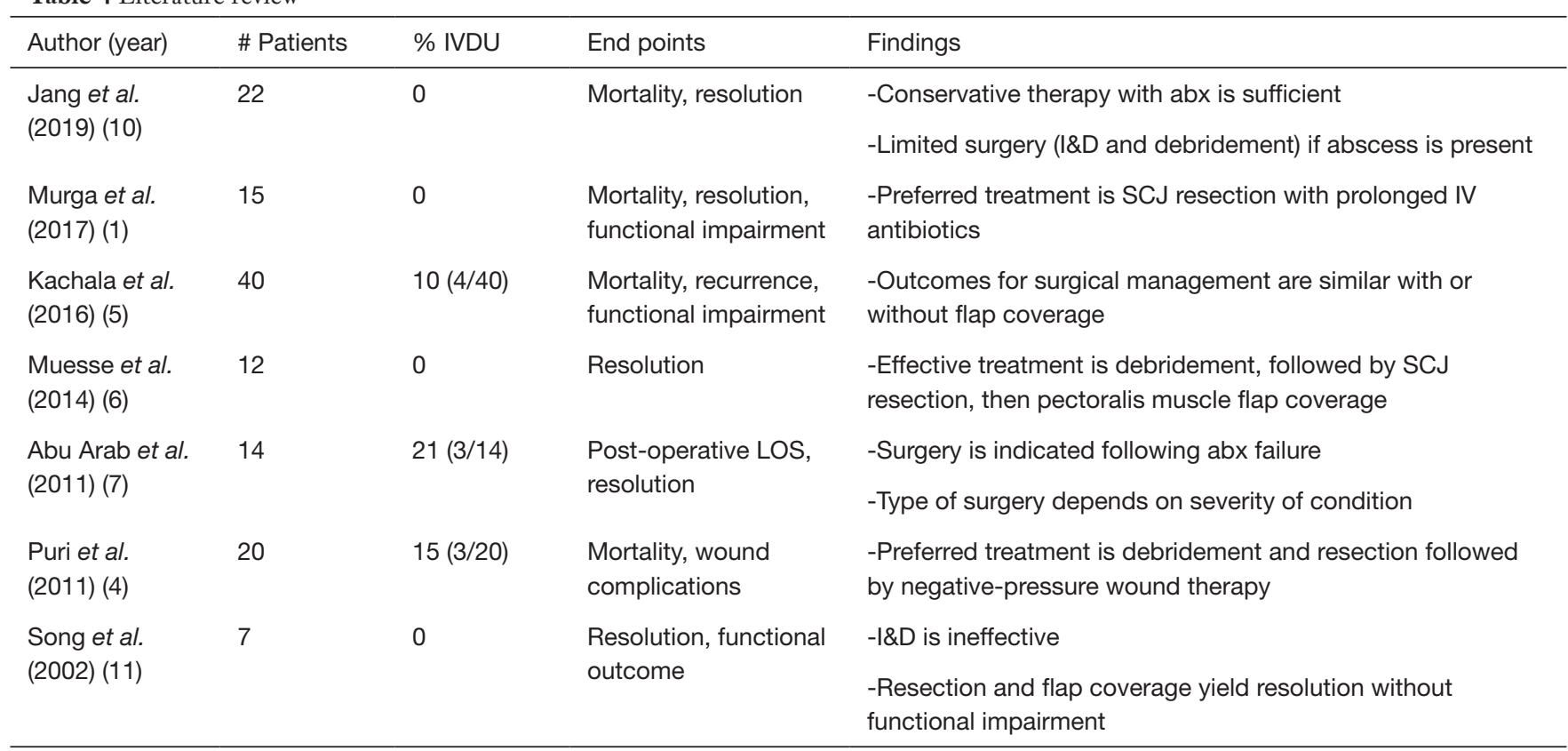

LOS, length of stay; abx, antibiotics; I\&D, incision and drainage; SCJ, sternoclavicular joint; IV, intravenous.

aggressive surgical management on initial presentation may prevent serious sequelae of SCJi. A retrospective study of seven patients conducted by Song et al. affirms our approach, advising formal joint resection should be undertaken in those patients who require $I \& D$, or fail initial medical management (11).

Limitations of this study include retrospective review that may have introduced selection bias, as well as decreased power from low sample size due to rarity of infection. The low sample size also prevented us from performing more intricate statistical testing, such as multivariate analysis to assess for other comorbid risk factors as confounders of antibiotic failure in those with DM2.

\section{Conclusions}

In conclusion, our study demonstrated no association between IVDU and either initial treatment planning or outcome of non-operative management. However, DM2 is associated with failure of non-operative treatment. Whereas previously proposed SCJi treatment algorithms have been guided by imaging findings and early response to antibiotics $(4,7)$, we suggest potentially incorporating DM2 into the decision-making algorithm, favoring medical management in the absence of DM2. Presence of this factor may indicate increased severity of infection, requiring more aggressive, primary operative management. Furthermore, we concur with prior studies that have recommended wide joint resection for SCJi resolution following failure of medical management. Ultimately, while the opioid epidemic brings on unique challenges in the treatment of IVDU patients and may skew our medical instincts, our study serves as a reminder that all-too-familiar comorbidities such as diabetes continue to compromise our patients' outcomes.

\section{Acknowledgments}

Poster presented at the Southern Thoracic Surgical Association (STSA) $66^{\text {th }}$ Annual Meeting in Marco Island, Florida on November $9^{\text {th }}, 2019$.

Funding: None.

\section{Footnote}

Reporting Checklist: The authors have completed the STROBE reporting checklist. Available at http://dx.doi. org/10.21037/jtd-20-1897

Data Sharing Statement: Available at http://dx.doi. org/10.21037/jtd-20-1897 
Conflicts of Interest: All authors have completed the ICMJE uniform disclosure form (available at http://dx.doi. org/10.21037/jtd-20-1897). The authors have no conflicts of interest to declare.

Etbical Statement: The authors are accountable for all aspects of the work in ensuring that questions related to the accuracy or integrity of any part of the work are appropriately investigated and resolved. The study was conducted in accordance with the Declaration of Helsinki (as revised in 2013). The study was approved by the Boston University School of Medicine and Boston Medical Center (BMC) Institutional Review Board (IRB) (No. FWA00000301) and individual consent for this retrospective analysis was waived.

Open Access Statement: This is an Open Access article distributed in accordance with the Creative Commons Attribution-NonCommercial-NoDerivs 4.0 International License (CC BY-NC-ND 4.0), which permits the noncommercial replication and distribution of the article with the strict proviso that no changes or edits are made and the original work is properly cited (including links to both the formal publication through the relevant DOI and the license). See: https://creativecommons.org/licenses/by-nc-nd/4.0/.

\section{References}

1. Murga A, Copeland H, Hargrove R, et al. Treatment for sternoclavicular joint infections: a multi-institutional study. J Thorac Dis 2017;9:1503-8.

2. Ross JJ, Shamsuddin H. Sternoclavicular septic arthritis: review of 180 cases. Medicine (Baltimore) 2004;83:139-48.

3. Yood RA, Goldenberg DL. Sternoclavicular joint arthritis. Arthritis Rheum 1980;23:232-9.

Cite this article as: Reddy SB, Mizelle J, Sterbling HM, Lin B, Litle VR, Suzuki K. Type 2 diabetes is associated with failure of non-operative treatment for sternoclavicular joint infection. J Thorac Dis 2020;12(10):5468-5474. doi: 10.21037/jtd-20-1897
4. Puri V, Meyers BF, Kreisel D, et al. Sternoclavicular joint infection: a comparison of two surgical approaches. Ann Thorac Surg 2011;91:257-61.

5. Kachala S.S., D'Souza D.M., Teixeira-Johnson L. et al. Surgical management of sternoclavicular joint infections. Ann Thorac Surg 2016;101:2155-60.

6. Muesse JL, Blackmon SH, Ellsworth WA, et al. Treatment of sternoclavicular joint osteomyelitis with debridement and delayed resection with muscle flap coverage improves outcomes. Surg Res Pract 2014;2014:747315.

7. Abu Arab W, Khadragui I, Echavé V, et al. Surgical management of sternoclavicular joint infection. Eur J Cardiothorac Surg 2011;40:630-4.

8. Hickton, DJ, Leary, ML. National Heroin Task Force final report and recommendations. U.S. Department of Justice and White House Office of National Drug Control Policy, Washington, DC, 2015.

9. Walley, AY, Paasche, M, Orlow, EC, et al. Acute care hospital utilization among medical inpatients discharged with a substance use disorder diagnosis. J Addict Med 2012;6:50-6.

10. Jang YR, Kim T, Kim MC, et al. Sternoclavicular septic arthritis caused by Staphylococcus aureus: excellent results from medical treatment and limited surgery. Infect Dis (Lond) 2019;51:694-700.

11. Song HK, Guy TS, Kaiser LR, et al. Current presentation and optimal surgical management of sternoclavicular joint infections. Ann Thorac Surg 2002;73:427-31.

12. Casqueiro J, Casqueiro J, Alves C. Infections in patients with diabetes mellitus: A review of pathogenesis. Indian J Endocrinol Metab 2012;16 Suppl 1:S27-S36.

13. Shah BR, Hux JE. Quantifying the risk of infectious diseases for people with diabetes. Diabetes Care 2003;26:510-3. 